Gynäk. Rdsch. 1966;3:256

\title{
Das Mammakarzinom bei jungen Mädchen
}

\begin{tabular}{|l|l|}
\hline M.B. & Close \\
\hline N.G. & Maximov \\
\hline
\end{tabular}

Surgery Section, Santa Rosa Memorial Hospital, Santa Rosa, Calif.

Das Mammakarzinom ist vor dem 20. Lebensjahr eine Kurio-sität. Nur 1,8\% der an Mammakarzinom erkrankten Frauen sind jünger als 30 Jahre.

Die kritische Betrachtung der Literatur bis 1955 ergab ledig-lich 9 gesicherte Fälle von Mammakarzinom, wobei diese Kinder 3 und 15 Jahre alt waren. Nach 1955 wurde nurmehr 1 Fall eines 1 ljährigen Mädchens bekannt. Darüber hinaus gibt es einige Be-richte von Brustkrebs bei Knaben, die alle tödlich endeten.

Die Autoren beschreiben zwei selbst beobachtete Fälle von Mammakarzinom. Bei beiden Mädchen war die Menarche bereits eingetreten, eines war 14, das andere 17 Jahre alt. Das eine Mäd chen starb 9 Monate nach der Mastektomie, wobei das auftretende Drüsenrezidiv röntgenbestrahlt und eine zytostatische Behandlung durchgeführt wurde. Das andere Mädchen starb 6 Monate nach der Mastektomie, wobei ebenfalls eine Röntgen- und zytostatische Therapie durchgeführt wurde. In beiden Fallen waren axilläre Lymphknoten bei der initialen Mastektomie von Karzinom be fallen. F. Friedrich

Originaltitel: Carcinoma of breast in young girls. 